\title{
Traduire
}

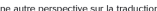

Revue française de la traduction

227 | 2012

Éco, socio, philo... \& co

\section{Traduire la littérature et les sciences humaines - conditions et obstacles, sous la direction de Gisèle Sapiro}

Françoise Wirth

\section{CpenEdition}

Journals

Édition électronique

URL : http://journals.openedition.org/traduire/502

DOI : $10.4000 /$ traduire.502

ISSN : 2272-9992

Éditeur

Société française des traducteurs

Édition imprimée

Date de publication : 15 décembre 2012

Pagination : 121-125

ISSN : 0395-773X

\section{Référence électronique}

Françoise Wirth, «Traduire la littérature et les sciences humaines - conditions et obstacles, sous la direction de Gisèle Sapiro », Traduire [En ligne], 227 | 2012, mis en ligne le 24 avril 2014, consulté le 22 septembre 2020. URL : http://journals.openedition.org/traduire/502 ; DOI : https://doi.org/10.4000/ traduire.502 


\title{
Traduire la littérature et les sciences humaines - conditions et obstacles, sous la direction de Gisèle Sapiro
}

\author{
Françoise Wirth
}

\section{RÉFÉRENCE}

Traduire la littérature et les sciences humaines - conditions et obstacles, sous la direction de Gisèle Sapiro, Collection Questions de culture, Département des études de la prospective et des statistiques, Ministère de la Culture et de la Communication, $14 €$. ISBN : 978-2-11-128148-6

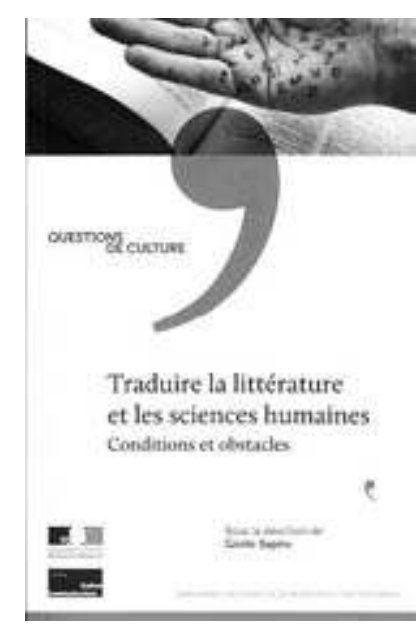

Une recension de cet ouvrage, paru tout récemment (septembre 2012), s'imposait dans ce numéro de Traduire consacré à la traduction des sciences sociales. Au travers de contributions d'auteurs différents réunis sous la direction - et avec la participation - de Gisèle Sapiro ${ }^{1}$, il brosse en effet un tableau des plus intéressants de la publication de 
traductions à partir du français et vers le français, en littérature et en sciences humaines.

2 Ce livre fait suite à une étude menée entre 2009 et 2011 au Centre européen de sociologie et science politique (CESSP), actuellement dirigé par Gisèle Sapiro, dans le cadre d'une convention avec le département d'études et de prospective du ministère de la Culture et de la Communication. 227 personnes ont été interrogées, éditeurs et responsables éditoriaux majoritairement, mais aussi traducteurs ou intermédiaires (agents littéraires notamment) dans cinq pays : la France, le Royaume-Uni, les ÉtatsUnis, les Pays-Bas et le Brésil. Le choix des pays est tout à fait pertinent : le RoyaumeUni et les États-Unis occupent incontestablement une position dominante sur le marché mondial, les Pays-Bas sont représentatifs d'un petit pays très actif au niveau éditorial et le Brésil, grand pays " émergent " d'Amérique du Sud, est en ascension sur le marché mondial de la traduction comme dans d'autres domaines et présente la caractéristique d'être un ancien pays colonisé entièrement émancipé de sa tutelle coloniale, au point d'avoir renversé le rapport de force avec celle-ci.

3 La première partie, consacrée à la présence du livre français à l'étranger, est composée de quatre chapitres présentant la situation dans chacun des pays de l'étude. Elle se situe sur un plan général et concerne plus particulièrement la littérature bien que de nombreuses observations soient également applicables aux sciences humaines. En revanche, la seconde partie - à l'exception de son premier chapitre qui traite des obstacles à l'importation des littératures étrangères en France - fait la part belle aux sciences humaines et sociales avec les deux chapitres qui suivent (chapitres 6 et 7). Enfin, la troisième partie analyse les aléas de trois parcours de traduction dans le domaine des sciences sociales: celui des œuvres du sociologue de langue allemande Norbert Elias, des représentants du pragmatisme américain tels que James Dewey et Charles Sanders Pierce, et des ouvrages de philosophie politique et morale de John Rawls.

4 L'angle de vue adopté pour cette étude est celui des obstacles à la circulation des œuvres et donc à la traduction. Dans une très intéressante introduction, Gisèle Sapiro dresse un tableau, tout à la fois général et concret, des obstacles d'ordre économique et culturel. La traduction est ainsi replacée dans le circuit éditorial et le lecteur en retire une idée claire des actions et décisions qui déterminent le choix des ouvrages qui seront traduits. Si certaines contingences sont bien connues, d'autres le sont moins. L'idée, par exemple, que les compétences linguistiques des éditeurs eux-mêmes influent sur ces choix est moins immédiate. Il paraît pourtant on ne peut plus évident qu'ils préfèrent lire les textes dans la langue d'origine avant de prendre la décision d'investir dans une traduction... ce qui restreint mécaniquement l'éventail des langues traduites. Du côté des sciences sociales, il est aussi intéressant de noter que les domaines suscitent un intérêt très varié suivant les pays : les Américains s'intéressent surtout à la philosophie ou à l'histoire, les Anglais et les Argentins plutôt à la psychanalyse qui n'enthousiasme pas du tout les Néerlandais, lesquels lui préfèrent les «neurosciences » (p. 51).

5 Dans la première partie orientée sur le rayonnement de la littérature française à l'étranger, certains éléments sont communs aux différents pays étudiés, la traduction étant souvent la première victime d'une recherche de limitation des coûts et de profits à court terme. On retrouve une dichotomie entre pôle de grande production (grandes maisons d'édition) et pôle de production restreinte (petits éditeurs plus ou moins 
spécialisés) doublée d'une opposition entre logique économique et logique symbolique ainsi que la prépondérance écrasante de la production anglo-américaine, conséquence directe de la position dominante des États-Unis dans l'espace culturel transnational.

Ce dernier point s'accompagne d'une marginalisation croissante du marché étatsunien de la traduction depuis les années 70 : les traductions y sont en perte de vitesse alors qu'elles ont augmenté partout ailleurs, n'y représentant que $3 \%$ des nouveautés contre une moyenne de $25 \%$ en France (chiffres de 2004, p. 62).

7 Parallèlement, le déclin des traductions du français est la conséquence logique de la perte d'influence de la culture française. Ce déclin est sensible aux Pays-Bas (depuis les années 70 avec une certaine stabilisation après 2000) comme au Brésil où la relation privilégiée qui existait autrefois avec l'Europe, et singulièrement avec la France, s'est distendue. En particulier, dans ce dernier pays, le Français a perdu son rôle de langue de traduction intermédiaire alors que, par le passé, de nombreuses traductions de langues « plus rares » étaient effectuées à partir de la traduction en français.

Comme on le sait, à la différence de ce que l'on observe dans les pays anglophones, en France, la traduction littéraire est un domaine éditorial bien établi, y compris parmi les grands éditeurs généralistes qui disposent pour la plupart de collections spécifiques. $\mathrm{Si}$, chez ceux-ci, les traductions de l'anglais sont prépondérantes, les plus petits éditeurs jouent la carte d'une plus grande diversité des langues traduites. Globalement, le nombre des traductions annuelles en français a doublé entre 1980 et 2002 (chiffres de l'Index Translationum de l'UNESCO), ce qui représente une hausse de $100 \%$, deux fois supérieure au taux d'augmentation de l'ensemble des traductions dans le monde ( $\mathrm{p}$. 210).

9 Et quels sont donc les éléments spécifiques de la traduction dans le domaine des sciences humaines et sociales?

10 Selon Sylvie Bosser, auteure du chapitre 6, ce domaine connaît des contraintes spécifiques souvent communes aux grandes maisons d'édition généralistes très présentes dans ce secteur et aux plus petites maisons d'édition dites « savantes ».

11 De manière peu surprenante, le coût est cité comme le principal obstacle à la traduction, avec au premier rang la rémunération du traducteur, mais aussi les droits payés à l'éditeur étranger cessionnaire et au directeur de collection. Dans ce domaine, plus que dans un autre (ouvrages souvent longs, lectorat plus restreint que pour la littérature), la traduction est un investissement qui ne s'amortit que sur le long terme. Compte tenu d'une conjoncture économique particulièrement difficile, la prudence est plus que jamais de rigueur dans le choix des ouvrages traduits.

12 Il semble aussi que la compétence des traducteurs soit un obstacle particulièrement important dans ce domaine puisque la traduction des sciences humaines et sociales mobilise des concepts, des notions et des représentations complexes n'ayant pas forcément d'équivalent dans la langue cible. On retrouve l'éternel dilemme entre confier la traduction à un spécialiste du domaine ne maitrisant pas nécessairement les finesses de la langue ou à un traducteur professionnel ne maitrisant pas nécessairement la complexité du sujet, problème parfois réglé par le travail en binôme entre un universitaire spécialiste du domaine et un traducteur professionnel. Il est d'ailleurs à noter qu'il existe une forte connivence entre maisons d'édition "savantes " et monde académique, les directeurs de collection de sciences humaines y étant 
souvent des universitaires, d'où des répercussions notables sur le choix des ouvrages à traduire.

13 La question du coût de la traduction est un problème que règlent différemment les petits éditeurs indépendants de sciences humaines auxquels est consacré le chapitre 7, rédigé par Sophie Noël. Ceux-ci ont en effet recours à des "ressources informelles " (l'un des sous-titres du chapitre) : ils se chargent eux-mêmes de la traduction (cas de maisons d'édition créées par des spécialistes d'une ou plusieurs langues) ou font du "bricolage", en faisant appel à des proches, à des bénévoles, à des traducteurs débutants "payés en deçà des tarifs en vigueur en échange de la formation et de l'expérience acquises» (p. 275). Il faut dire à leur décharge qu'il s'agit d'éditeurs engagés à qui l'on doit souvent la découverte d'auteurs de langues diverses, car ils sont prêts, en acceptant eux-mêmes de travailler dans des conditions économiques difficiles, à prendre le risque de publier des auteurs traduits, susceptibles de leur permettre d'accumuler un capital symbolique et de s'affirmer sur le marché. C'est ainsi qu'avec une importante part de traductions inscrite à leur catalogue (65\% par exemple pour L'Éclat), de petits éditeurs misent fortement sur la traduction, à l'inverse du secteur général des sciences humaines qui connaît un fort déclin des traductions $(4,5 \%$ seulement en 2000). L'article s'accompagne de tableaux présentant la part des titres traduits de différentes langues vers le français dans les catalogues de petits éditeurs de sciences humaines ${ }^{2}$.

Soulignons que la plupart des contributions comprennent une partie retraçant l'histoire de la traduction dans les pays concernés et sont illustrées par des graphiques très parlants détaillant l'évolution dans le temps de la part des ouvrages traduits dans la production totale, ainsi que l'évolution de la répartition des langues sources selon les pays. Certains sont également accompagnés d'encadrés, permettant un «zoom » sur des cas où des aspects particuliers (citons, par exemple, l'encadré de Cécile Balayer sur le rôle des libraires dans la valorisation de la littérature traduite, chapitre 5, p. 225).

Malgré un tableau d'ensemble peu réjouissant, mettant en évidence la tendance croissante à privilégier la logique économique, peu favorable à la traduction, notons que cet ouvrage très bien documenté ne donne aucunement un sentiment de catastrophisme. Il insiste au contraire sur la vivacité et le dynamisme d'acteurs engagés qui, du côté du pôle de production restreinte, continuent un peu partout à privilégier la valeur intellectuelle et à trouver des manières viables de le faire. Il ressort de l'ensemble de l'ouvrage que les mécanismes de subventions à la traduction et à la diffusion des ouvrages français accordées par le Centre national du livre (CNL) ou par les ministères des Affaires étrangères et de la Culture sont appréciés tant en France qu'à l'étranger et constituent une béquille indispensable. Les traducteurs, même si l'on peut considérer que la reconnaissance de leur travail est loin d'être parfaite en France, se heurtent à des problèmes encore plus grands ailleurs. Dans les pays anglophones par exemple, les traductions sont peu valorisées et au prétexte d'un désamour systématique des lecteurs pour les traductions en général, relevant peut-être " d'une prophétie auto réalisatrice » (p. 68), on tente souvent de les rendre aussi invisibles que possible. La reconnaissance juridique et fiscale, voire symbolique, dont jouit le traducteur d'édition en France (mention du nom du traducteur sur la publication et dans la bibliographie nationale) peut paraître enviable à des collègues d'autres pays. Il est clair que le coût de la traduction et donc la rémunération des traducteurs sont considérés par la plupart des acteurs comme un obstacle à la diffusion d'ouvrages 
traduits. Comme souvent, on a là une double contrainte, les éditeurs reconnaissant la difficulté du travail du traducteur (notamment dans le domaine des sciences sociales, où ils se plaignent du trop petit nombre des personnes disposant des compétences nécessaires), tout en reculant devant la nécessité de rémunérer correctement ce travail au vu des difficultés économiques du secteur. À ce propos, il est étonnant que ne soit pas davantage abordé dans cet ouvrage les nouvelles perturbations que risque d'entraîner l'évolution de l'édition vers le numérique, brièvement évoquée dans l'article sur le Brésil. Il est vrai qu'il s'agit davantage du bilan de la situation présente.

C'est en tout cas une étude qui fourmille d'informations et de remarques éclairantes, que tout traducteur, travaillant ou non dans les domaines concernés, lira avec grand profit.

\section{NOTES}

1. Voir aussi son article «La circulation des sciences humaines et sociales en traduction : enjeux et obstacles à l'heure de la globalisation » en page 7 de ce numéro.

2. Il est toutefois regrettable que ces tableaux ne mentionnent aucun nom de traducteur. 